
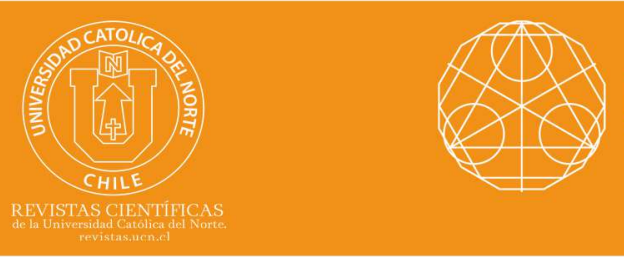

\title{
On the upper geodetic global domination number of a graph
}

\author{
X. Lenin Xaviour ${ }^{1}$ (1) orcid.org/0000-0001-9263-6492 \\ S. Robinson Chellathurai ${ }^{2}$ \\ ManonmaniamSundaranarUniversity,ScottChristian College, Dept of Mathematics, Nagercoil,TN, India. \\ ${ }^{1 \rrbracket}$ leninxaviour93@gmail.com ; ${ }^{\circledR}$ robinchel@rediffmail.com
}

Received: November 2019 | Accepted: April 2020

\section{Abstract:}

$A$ set $S$ of vertices in a connected graph $G=(V, E)$ is called a geodetic set if every vertex not in $S$ lies on a shortest path between two vertices from $S$. A set $D$ of vertices in $G$ is called a dominating set of $G$ if every vertex not in $D$ has at least one neighbor in $D$. A set $D$ is called a global dominating set in $G$ if $S$ is a dominating set of both $G$ and $\bar{G}$. A set $S$ is called a geodetic global dominating set of $G$ if $S$ is both geodetic and global dominating set of $G$. A geodetic global dominating set $S$ in $G$ is called a minimal geodetic global dominating set if no proper subset of $S$ is itself a geodetic global dominating set in $G$. The maximum cardinality of a minimal geodetic global dominating set in $G$ is the upper geodetic global domination number $\bar{Y}_{g}^{+}(G)$ of $G$. In this paper, the upper geodetic global domination number of certain connected graphs are determined and some of the general properties are studied. It is proved that for all positive integers $a, b, p$ where $3 \leq a \leq b<p$, there exists a connected graph $G$ such that $\bar{Y} g(G)=a, \bar{Y}_{g}^{+}(G)=b$ and $|V(G)|=p$.

Keywords: Geodetic set; Dominating set; Geodetic domination; Geodetic global domination; Upper geodetic global domination number.

MSC (2020): 05C12, 05C75.

\section{Cite this article as (IEEE citation style):}

X. L. Xaviour and S. R. Chellathurai, "On the upper geodetic global domination number of a graph", Proyecciones (Antofagasta, On line), vol. 39, no. 6, pp. 1627-1646, Dec. 2020, doi: 10.22199/issn.0717-6279-2020-06-0097.

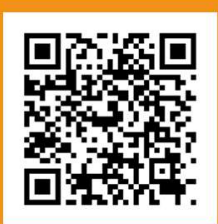

Article copyright: (C) $2020 \mathrm{X}$. Lenin Xaviour and S. Robinson Chellathurai. This is an open access article distributed under the terms of the Creative Commons License, which permits unrestricted use and distribution provided the original author and source are credited.

(cc) BY 


\section{Introduction}

Throughout this paper, graph $G=(V, E)$ we mean a simple, finite, connected, undirected graph with neither loops nor multiple edges. The order $|V|$ and size $|E|$ of $G$ are denoted by $p$ and $q$ respectively. For graph theoretic terminology we refer to West[9]. A graph $G$ is said to be totally disconnected if $|E(G)|=0$. The open neighborhood of any vertex $v$ in $G$ is $N(v)=\{x: x v \in E(G)\}$ and closed neighborhood of a vertex $v$ in $G$ is $N[v]=N(v) \cup\{v\}$. The degree of a vertex in the graph $G$ is denoted by $\operatorname{deg}(v)$ and the maximum degree (minimum degree) in the graph $G$ is denoted by $\triangle(G)(\delta(G))$. For a set $S \subseteq V(G)$ the open (closed) neighborhood $N(S)(N[S])$ in $G$ is defined as $N(S)=\bigcup_{v \in S} N(v)\left(N[S]=\bigcup_{v \in S} N[v]\right)$. We write $K_{p}, P_{p}, C_{p}, W_{p}$ and $F_{p}$ for the complete graph. a path graph, a cycle graph, a wheel graph and a fan graph of order $p$, respectively.

If $G$ is a connected graph the distance $d(x, y)$ is the length of a shortest $x-y$ path in $G$. The diameter is defined by $\operatorname{diam}(G)=\max _{x, y \in V(G)} d(x, y)$. Two vertices $u$ and $v$ are said to be antipodal vertices if $d(u, v)=\operatorname{diam}(G)$. If $e=\{u, v\}$ is an edge of a graph $G$ with $\operatorname{deg}(u)=1$ and $\operatorname{deg}(v)>1$, then we call $e$ a pendant edge, $u$ a pendant vertex and $v$ a support vertex. A vertex $v$ of $G$ is said to be an extreme vertex if the subgraph induced by its neighborhood is complete. The set of all extreme vertices is denoted by $\operatorname{Ext}(G)$. A vertex $v$ is said to be a full vertex if $v$ is adjacent to all other vertices in $G$, that is, $\operatorname{deg}(v)=p-1$. The set of all full vertices is denoted by $F x(G)$. The girth of a graph $G$ is the length of a shortest cycle contained in $G$ and is denoted by $c(G)$. An acyclic connected graph is called a tree. An $x-y$ path of length $d(x, y)$ is called geodesic. A vertex $v$ is said to lie on a geodesic $P$ if $v$ is an internal vertex of $P$. The closed interval consists of $x, y$ and all vertices lying on some $x-y$ geodesic of $G$ and for a non empty set $S \subseteq V(G), I[S]=\bigcup_{x, y \in S} I[x, y]$.

A set $S \subseteq V(G)$ in a connected graph $G$ is a geodetic set of $G$ if $I[S]=V(G)$. The geodetic number of $G$, denoted by $g(G)$, is the minimum cardinality of a geodetic set of $G$. A geodetic set $S$ in $G$ is a minimal geodetic set in $G$ if no proper subset of $S$ is a geodetic set of $G$. The maximal cardinality of minimal geodetic set of $G$ is the upper geodetic number $g^{+}(G)$ of $G$. Various concepts inspired by geodetic sets and minimal geodetic sets are introduced in $[1,2,3]$.

A set $S \subseteq V(G)$ in a graph $G$ is a dominating set of $G$ if for every vertex $v$ in $V-S$, there exists a vertex $u$ in $S$ such that $v$ is adjacent to $u$. The 
domination number of $G$, denoted by $\gamma(G)$, is the minimum cardinality of a dominating set of $G[5]$.

The concept of geodetic dominating sets and minimal geodetic dominating sets are introduced and studied in $[4,6]$. A set $S \subseteq V(G)$ is a geodetic dominating set of $G$ if $S$ is both geodetic and dominating set of $G$. The geodetic domination number, denoted by $\gamma_{g}(G)$, is the minimum cardinality of a geodetic dominating set of $G[4]$. A geodetic dominating set of $G$ is called a minimal geodetic dominating set of $G$ if no proper subset of $S$ is a geodetic dominating set of $G$. The upper geodetic domination number, denoted by $\gamma_{g}^{+}(G)$, is the minimum cardinality of a minimal geodetic dominating set of $G[6]$.

The complements $\bar{G}=(\bar{V}, \bar{E})$ of $G=(V, E)$ is the graph with vertex set $\bar{V}=\{\bar{v}: v \in V\}$ and $\bar{E}=\{(\bar{u}, \bar{v}): \bar{u}, \bar{v} \in \bar{V}, u=v$ and $(u, v) \notin E\}$.

A set $S \subseteq V(G)$ is called a global dominating set of $G$ if it is a dominating set of both $G$ and $\bar{G}$ [8]. The global domination number of $G$, denoted by $\bar{\gamma}(G)$, is the minimum cardinality of a geodetic global dominating set of $G$.

In [7], authors studied geodetic global dominaiton number in graphs which is defined as follows:

Definition 1.1 Let $G=(V, E)$ be connected with at least two vertices. A subset $S \subseteq V(G)$ is called a geodetic global dominating set of $G$ if $S$ is both geodetic and global dominating set of $G$. The geodetic global domination number denoted by $\bar{\gamma}_{g}(G)$ is the minimum cardinality of a geodetic global dominating set of $G$ and the geodetic global dominating set with cardinality $\bar{\gamma}_{g}(G)$ is called the $\bar{\gamma}_{g}$-set of $G$ or $\bar{\gamma}_{g}(G)$-set.

\section{Some useful known results}

Theorem 2.1. [7] Let $G$ be connected graph. Then $\operatorname{Ext}(G) \subseteq S$ for all geodetic global dominating sets $S$ in $G$.

Theorem 2.2. [7] Let $G$ be a connected graph. Then $F x(G) \subseteq S$ for all geodetic global dominating sets $S$ in $G$.

Theorem 2.3. [7] Let $G$ be a connected graph of order $p$ then

(i) $\bar{\gamma}_{g}(G)=2$ if and only if $G=K_{2}$ or there exists a geodetic set $S=\{u, v\}$ such that $d(u, v)=3$.

(ii) $\bar{\gamma}_{g}(G)=p$ if and only if $G$ contains only the extreme and full vertices. 
Theorem 2.4. [7] Let $G$ be a connected graph with $F x(G)=\phi$. Then, $\bar{\gamma}_{g}(G)=p-1$ if and only if $G \simeq C_{4}$.

Theorem 2.5. [7] For the complete bipartite graph $K_{p, q}(p, q \geq 2)$,

$$
\bar{\gamma}_{g}\left(K_{p, q}\right)=\left\{\begin{array}{cc}
\min \{p, q\}+1 & \text { if } 2 \leq p, q \leq 3 \\
4 & \text { if } p, q \geq 4
\end{array}\right.
$$

\section{Upper Geodetic Global Domination Number of a Graph}

Definition 3.1. Let $G=(V, E)$ be a connected graph of order $p \geq 2$. A geodetic global dominating set $S$ in $G$ is called a minimal geodetic global dominating set in $G$ if no proper subset of $S$ itself a geodetic global dominating set in $G$. The upper geodetic global domination number $\bar{\gamma}_{g}^{+}(G)$ is the maximum cardinality of a minimal geodetic global dominating set in $G$. A minimal geodetic global dominating set with cardinality $\bar{\gamma}_{g}^{+}(G)$ is called a $\bar{\gamma}_{g}^{+}$-set of $G$ or $\bar{\gamma}_{g}^{+}(G)$-set.

We illustrate this definition by an example.

Example 3.2. For the graph $G$ in Figure 3.1, it is easy to see that $S=$ $\left\{v_{1}, v_{6}\right\}$ is a minimum geodetic dominating set of $G$ and so $\gamma_{g}(G)=2$. Since, $\operatorname{diam}(G)=2$, it is clear that no 2-element subset of vertices is a geodetic global dominating set of $G$. It is easily verified that $S_{1}=$ $\left\{v_{1}, v_{2}, v_{6}\right\}$ and $S_{2}=\left\{v_{1} \cdot v_{3}, v_{6}\right\}$ are the only two minimum geodetic global dominating sets and so $\bar{\gamma}_{g}(G)=3$. It is also easily verified that $S_{1}^{\prime}=$ $\left\{v_{1}, v_{2}, v_{3}, v_{4}, v_{5}\right\}$ and $S_{2}^{\prime}=\left\{v_{2}, v_{3}, v_{4}, v_{5}, v_{6}\right\}$ are the only two minimal geodetic global dominating sets of order 5 . Since there is no minimal geodetic global dominating set of order greater than $5, \bar{\gamma}_{g}^{+}(G)=5$. 


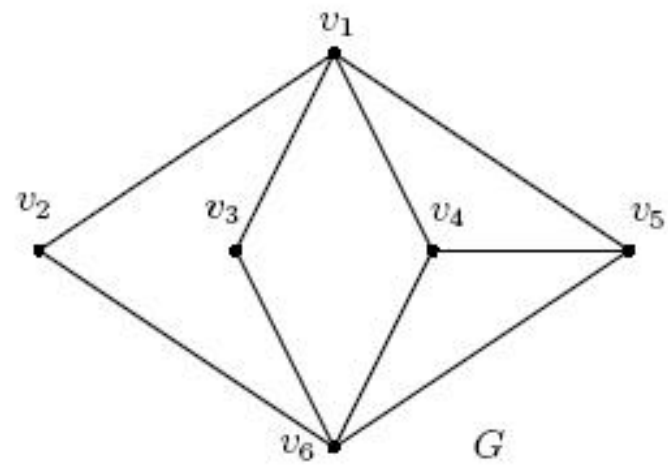

Figure 3.1

Remark 3.3. Every minimum geodetic global dominating set is a minimal geodetic global dominating set of $G$, and the converse need not be true. For the graph $G$ in Figure 3.1, $S_{1}^{\prime}=\left\{v_{1}, v_{2}, v_{3}, v_{4}, v_{5}\right\}$ is a minimal geodetic global dominating set of $G$ and not a minimum geodetic global dominating set of $G$. Thus, the geodetic global domination number and the upper geodetic global domination number are different.

Theorem 3.4. Let $G$ be a connected graph of order $p$. Then $2 \leq \bar{\gamma}_{g}(G) \leq$ $\bar{\gamma}_{g}^{+}(G) \leq p$.

Proof. Any geodetic global dominating set has atleast two vertices and so $\bar{\gamma}_{g}(G) \geq 2$. The inequality $\bar{\gamma}_{g}(G) \leq \bar{\gamma}_{g}^{+}(G)$ follows from Remark 3.3. Also, all vertices of $G$ form a geodetic global dominating set of $G$ and therefore, $\bar{\gamma}_{g}^{+}(G) \leq p$. Thus, $2 \leq \bar{\gamma}_{g}(G) \leq \bar{\gamma}_{g}^{+}(G) \leq p$.

Remark 3.5. The bound in Theorem 3.4 are sharp. For the path graph $P_{4}, \bar{\gamma}_{g}\left(P_{4}\right)=2$. For any bistar graph $G$ of order at least 2 , it is clear that the set of all end vertices is the unique minimum geodetic global dominating set of $G$ and so $\bar{\gamma}_{g}(G)=\bar{\gamma}_{g}^{+}(G)$. All the inequalities in Theorem 3.4 can be strict. For the graph $G$ in Figure 3.1, $\bar{\gamma}_{g}(G)=3, \bar{\gamma}_{g}^{+}(G)=5$ and $p=6$. Thus, $2<\bar{\gamma}_{g}(G)<\bar{\gamma}_{g}^{+}(G)<p$.

Corollary 3.6. Let $G$ be a connected graph of order $p$. If $\bar{\gamma}_{g}^{+}(G)=2$, then $\bar{\gamma}_{g}(G)=2$. 
Proof. This follows from Theorem 3.4

Remark 3.7. The converse of the Corollary 3.6 need not be true. For the graph $G$ given in Figure 3.2, we note that $\bar{\gamma}_{g}(G)=2$ and $\bar{\gamma}_{g}^{+}(G)=4$. Thus, $\bar{\gamma}_{g}^{+}(G)=2$.

Corollary 3.8. Let $G$ be a connected graph of order $p$. If $\bar{\gamma}_{g}(G)=p$, then $\bar{\gamma}_{g}^{+}(G)=p$.

Proof. This follows from Theorem 3.4.

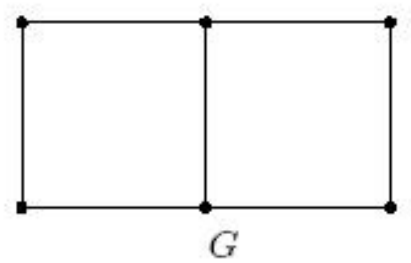

Figure 3.2

The following theorem characterizes the graphs for which the upper geodetic global domination number is 2 .

Theorem 3.9. Let $G$ be a connected graph of order $p \geq 2$. Then $\bar{\gamma}_{g}^{+}(G)=$ 2 if and only if $G=K_{2}$ or $G$ has a geodetic set $S=\{x, y\}$ with $x, y \in$ $\operatorname{Ext}(G)$ and $d(x, y)=3$.

Proof. Suppose $\bar{\gamma}_{g}^{+}(G)=2$. Then by Corollary 3.6, $\bar{\gamma}_{g}(G)=2$. By Theorem 2.3, we have $G=K_{2}$ or there is a geodetic set $S=\{x, y\}$ with $d(x, y)=3$. If $G=K_{2}$, then the proof is over. Suppose that $d(x, y)=3$. Then for each $u$ in $V(G)-S, u$ is adjacent to either $x$ or $y$. Without loss of generality, we assume $u x \in E(G)$. Suppose on the contrary $x \notin$ $\operatorname{Ext}(G)$. Then there exists a vertex $v$ such that $u, v \in N(x)$ with $d(u, v)=$ 2. Consider, $D=N(x) \cup\{y\}$. Clearly, $|D| \geq 3$. Let $z \in V(G)-D$. If $z=x$, then $z$ lies on a $u-v$ geodesic in $G$ and so that $z \in I[D]$ and $z \in N[D]$. Also, $d(z, y)=3, z \in N_{\bar{G}}[D]$, implies $D$ is a geodetic global dominating set in $G$. Suppose $z=x$. Since, $z \in I[x, y]$, there exists $w, t$ in $V(G)$ such that $z$ lies on the $x-y$ geodesic $\{x, w, t, y\}$. It follows that $z \in I[D]$ and $z \in I[D]$. If $N(z) \subseteq D$, then there exists a set $T \subseteq D$ such 
that $T$ is a minimal geodetic global dominating set of cardinality greater than or equal to 3 , a contradiction. Therefore, $N(z) D$ for all $z \notin D$, implies $z \in N_{\bar{G}}[D]$. Thus both the cases, $D$ is a geodetic global dominating set in $G$. Let $W \subseteq D$ be a minimal geodetic global dominating set in $G$. Since, $y \notin N[N(x)], y \in W$. Also if $|W \cap N(x)|=1$, then $x \notin I[W]$, a contradiction. Thus, $|W \cap N(x)| \geq 2$ implies $\bar{\gamma}_{g}^{+}(G) \geq|W| \geq 3$, a contradiction. Hence $<N[x]>$ is complete and $x \in \operatorname{Ext}(G)$. Similarly, we can prove that $y \in \operatorname{Ext}(G)$. Conversely, if $G=K_{2}$, then $\bar{\gamma}_{g}^{+}(G)=2$. Now, let $G$ have a geodetic set $S=\{x, y\}$ with $d(x, y)=3$ and here the induced subgraphs $<N[x]>$ and $<N[y]>$ are complete. Then $\operatorname{Ext}(G)=\{x, y\}$ is the unique minimal geodetic global dominating set in $G$ and so $\bar{\gamma}_{g}^{+}(G)=2$.

Theorem 3.10. Let $G$ be a connected graph of order $p$. Then,

(i) Every minimal geodetic global dominating sets of $G$ contains all the extreme vertices in $G$.

(ii) Every minimal geodetic global dominating sets of $G$ contains its full vertices.

(iii) If $G$ has a unique minimal geodetic global dominating set, then $\bar{\gamma}_{g}(G)=$ $\bar{\gamma}_{g}^{+}(G)$.

Proof. (i) and (ii) from Theorem 2.1 and Theorem 2.2

(iii) Since any minimal geodetic global dominating sets are nothing but the minimum geodetic global dominating sets, $\bar{\gamma}_{g}(G)=\bar{\gamma}_{g}^{+}(G)$.

The following Theorem characterizes the graphs for which the upper geodetic global domination number is $p$.

Theorem 3.11. Let $G$ be a connected graph of order $p$. Then $\bar{\gamma}_{g}(G)=p$ if and only if $\bar{\gamma}_{g}^{+}(G)=p$.

Proof. If $\bar{\gamma}_{g}(G)=p$, then by Corollary $3.8, \bar{\gamma}_{g}^{+}(G)=p$. Conversely, if $\bar{\gamma}_{g}^{+}(G)=p$. Then, the set of all vertices of $G$ is the unique minimal geodetic global dominating set of $G$. It follows that $G$ contains no proper geodetic global dominating sets and so the set of all vertices is the minimum geodetic global dominating set of $G$. Hence, $\bar{\gamma}_{g}(G)=p$.

Corollary 3.12. For the complete graph $K_{p}(p \geq 2), \bar{\gamma}_{g}\left(K_{p}\right)=\bar{\gamma}_{g}^{+}\left(K_{p}\right)=$ $p$. 
Proof. This follows from Theorem 3.11

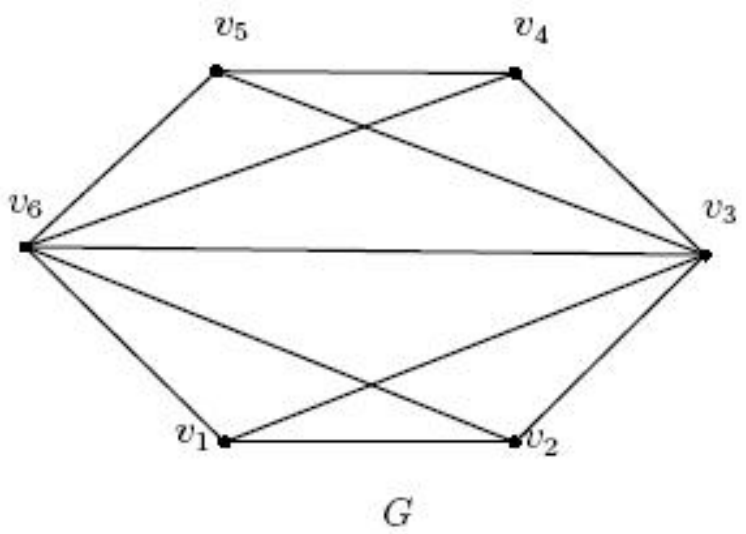

Fiqure 3.3

Remark 3.13. The converse of Corollary 3.12 need not be true. For the graph $G$ in Figure 3.3, the set $S=\left\{v_{1}, v_{2}, v_{3}, v_{4}, v_{5}, v_{6}\right\}$ is the unique minimum geodetic global dominating set of $G$ and so $\bar{\gamma}_{g}(G)=6$. Hence, by Theorem 3.11, $\bar{\gamma}_{g}^{+}(G)=6$.

This examples show that there are non-complete graphs $G$ of order $p$ with $\bar{\gamma}_{g}^{+}(G)=\bar{\gamma}_{g}(G)=p$.

Theorem 3.14. If $G$ is a connected graph of order $p$ with $\bar{\gamma}_{g}(G)=p-1$, then $\bar{\gamma}_{g}^{+}(G)=p-1$.

Proof. Let $\bar{\gamma}_{g}(G)=p-1$. Then either $\bar{\gamma}_{g}^{+}(G)=p$ or $\bar{\gamma}_{g}^{+}(G)=p-1$ by applying Theorem 3.4. If $\bar{\gamma}_{g}^{+}(G)=p$, then by Theorem 3.11, $\bar{\gamma}_{g}(G)=p$, which contradicts our hypothesis. Hence, $\bar{\gamma}_{g}^{+}(G)=p-1$.

Remark 3.15. The converse of Theorem 3.14 need not be true. For the graph in Figure 3.4, the set $S=\left\{v_{1}, v_{2}, v_{3}\right\}$ is a minimum geodetic global dominating set of $G$ and so $\bar{\gamma}_{g}(G)=3$. Also, it is easily seen that the set $S^{\prime}=\left\{v_{1}, v_{3}, v_{4}, v_{5}\right\}$ is a minimal geodetic global dominating set and so $\bar{\gamma}_{g}^{+}(G)=4=p-1$. But $\bar{\gamma}_{g}(G)=3=p-1$. 


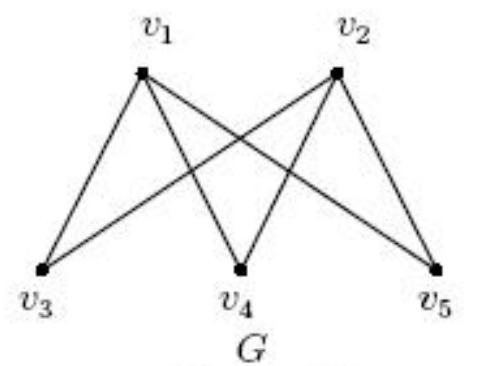

Figure 3.4

Theorem 3.16. Let $G$ be a connected graph with cut vertices and let $S$ be a minimal geodetic global dominating set of $G$. If $v$ is a cut vertex of $G$ then every component of $G-v$ contains an element of $S$.

Proof. Suppose that there is a component $H$ of $G-v$ such that $H$ contains no vertex of $S$. Let $u$ be a vertex in $H$. Since, $S$ is a minimal geodetic global dominating set of $G$, there exist vertices $x, y \in S$ such that $u$ lies on some $x-y$ geodesic path $P: x=u_{0}, u_{1}, \ldots, u, \ldots, u_{l}=y$ in $G$. Let $P_{1}$ be the $x-u$ subpath of $P$ and $P_{2}$ be the $u-y$ subpath of $P$. Since, $v$ is a cut vertex of $G$, both $P_{1}$ and $P_{2}$ contains $v$ so that $P$ is not a path, which is a contradiction. Thus, every component of $G-v$ contains an element of $S$.

begincor Let $G$ be a connected graph with cut vertices and let $S$ be a minimal geodetic global dominating set of $G$. Then every branch of $G$ contains an element of $S$.

Theorem 3.17. For the complete bipartite graph $K_{p, q}(p, q \geq 2), \bar{\gamma}_{g}^{+}\left(K_{p, q}\right)=$ $\max \{p, q\}+1$.

Proof. Let $G=K_{p, q}$ where $p, q \geq 2$. Let $X=\left\{x_{1}, x_{2}, \ldots, x_{p}\right\}$ and $Y=\left\{y_{1}, y_{2}, \ldots, y_{q}\right\}$ be the bipartition of $G$. Without loss of generality, we assume $q \leq p$. 
case (i) $q<p$, then, $|X|>|Y|$. Since, each $y_{i} \in I\left[x_{j}, x_{k}\right] \subseteq I[X]$ and $y_{i} \in N\left[x_{j}\right] \subseteq N[x], X$ is a geodetic dominating set of $G$. In $G,\langle X>$ and $\langle Y\rangle$ are disjoint complete graphs. Therefore, $X$ is not a dominating set of $\bar{G}$. Now, let $S=X \cup\left\{y_{i}\right\}, i=1,2, \ldots, q$. Clearly, $S$ is a dominating set of $\bar{G}$. Since, $I[S]=V(G)$ and $N[S]=V(G)$, that $S$ is a geodetic global dominating set of $G$. Now, we show that $S$ is a minimal geodetic global dominating set of $G$. Suppose there exists a geodetic global dominating set $S_{1}$ of $G$ such that $S_{1} \subset S$. Suppose there exists a vertex $x_{j} \in S$ such that $x_{j} \notin S_{1}$. Clearly, the vertex $x_{j}$ does not lie on any geodesic path joining a pair of vertices in $S_{1}$. That is, $I\left[S_{1}\right]=V(G)$. Thus, $S_{1}$ is not a geodetic global dominating set of $G$. If $y_{i} \notin S_{1}$, then $N\left[S_{1}\right]=V(\bar{G})$, implies $S_{1}$ is not a geodetic global dominating set of $G$. Both ways, we obtained a contradiction. Therefore, $S$ is a minimal geodetic global dominating set of $G$. Hence, $\bar{\gamma}_{g}^{+}(G) \geq|S|=p+1$. Now, we went to show that $\bar{\gamma}_{g}^{+}(G)=p+1$. Suppose, there exists a minimal geodetic global dominating set $S_{2}$ of $G$ such that $\left|S_{2}\right|>p+1$. Since, $X \cup\left\{y_{i}\right\}$ is a minimal geodetic global dominating set of $G$ and $\left|S_{2}\right|>\left|X \cup\left\{y_{i}\right\}\right|, S_{2}$ contains atleast two vertices from $Y$, say $y_{i}, y_{k}$. Then, clearly $S_{2}-\left\{y_{i}\right\}$ is a geodetic global dominating set of $G$, which is a contradiction. Therefore, $\bar{\gamma}_{g}^{+}(G)=p+1$.

case (ii) If $p=q$, then $|X|=|Y|$. Using a similar argument as in case (i), it can be show that $\bar{\gamma}_{g}^{+}(G)=q+1=p+1$. Hence, $\bar{\gamma}_{g}^{+}(G)=\max \{p, q\}+1$.

Theorem 3.18. For the path graph $P_{p},(p \geq 4), \bar{\gamma}_{g}^{+}\left(P_{p}\right)=\left\lceil\frac{p}{2}\right\rceil$.

Proof. Let $P_{p}: v_{1}, v_{2}, \ldots v_{p}$ be a path of order $p \geq 4$ in which $v_{i} v_{j} \in$ $E\left(P_{p}\right)$ if and only if $2 \leq j=i+1 \leq p$. We prove this theorem by considering two cases.

Case (i) $p$ is odd. Let $S=\left\{v_{1}, v_{3}, v_{5}, \ldots, v_{p-2}, v_{p}\right\}$. Clearly $S$ is a geodetic global dominating set in $P_{p}$ with maximum cardinality. If we remove any vertex from $S$, then $I[S]=V(G)$ implies, $S$ is not a geodetic global dominating set of $P_{p}$. Therefore, $\bar{\gamma}_{g}^{+}\left(P_{p}\right)=|S|=\left\lceil\frac{p}{2}\right\rceil$.

Case (ii) $p$ is even. Consider $S^{\prime}=\left\{v_{1}, v_{3}, v_{5}, \ldots, v_{p-3}, v_{p}\right\}$. It is straight forward that $S^{\prime}$ is a minimal geodetic global dominating set in $P_{p}$, with maximum cardinality. Thus, $\bar{\gamma}_{g}^{+}\left(P_{p}\right)=|S|=\left\lceil\frac{p}{2}\right\rceil$. Hence, both the cases, $\bar{\gamma}_{g}^{+}\left(P_{p}\right)=\left\lceil\frac{p}{2}\right\rceil$. 
Theorem 3.19. For the cycle $C_{p}(p \geq 3), \bar{\gamma}_{g}^{+}\left(C_{p}\right)=\left\{\begin{array}{cc}3 & \text { if } p=3,4,5 \\ \left\lfloor\frac{p}{2}\right\rfloor & \text { if } p \geq 6\end{array}\right.$

Proof. Let $C_{p}: v_{1}, v_{2}, \ldots v_{p}, v_{1}$ be a cycle of order $p \geq 3$ in which $v_{i} v_{j} \in E\left(C_{p}\right)$ if and only if $j \equiv i+1(\bmod p)$. For $p=3, C_{3} \simeq K_{3}$. Then by Corollary 3.12, $\bar{\gamma}_{g}^{+}\left(K_{3}\right)=\bar{\gamma}_{g}^{+}\left(C_{3}\right)=3$. If $p=4$, then by Theorem 2.4, $\bar{\gamma}_{g}\left(C_{4}\right)=4-1$. Therefore, by Theorem $3.14, \bar{\gamma}_{g}^{+}\left(C_{4}\right)=4-1=3$.

Now let $p \geq 5$ and let $V\left(C_{p}\right)=\left\{v_{1}, v_{2}, v_{3}, \ldots, v_{p}\right\}$. For $p=5$, take $S=\left\{v_{1}, v_{3}, v_{5}\right\}$. Clearly, $S$ is a minimal geodetic global dominating set of $C_{5}$ and no minimal geodetic global dominating set exists with cardinality greater than $|S|$. Therefore, $\bar{\gamma}_{g}^{+}\left(C_{5}\right)=3$. For $p \geq 6$, we consider two cases:

Case (i) $p$ is even. Let $p=2 n$. It is clear that $S=\left\{v_{1}, v_{3}, v_{5}, . ., v_{2 n-3}, v_{2 n-1}\right\}$ is a minimal geodetic global dominating set of $C_{2 n}$ and so $\bar{\gamma}_{g}^{+}\left(C_{2 n}\right) \geq|S|=$ $\frac{p}{2}=\left\lfloor\frac{p}{2}\right\rfloor$. Now we show that $\bar{\gamma}_{g}^{+}\left(C_{2 n}\right) \leq\left\lfloor\frac{p}{2}\right\rfloor$. Suppose there exists a minimal geodetic global dominating set $S_{1}$ such that $\left|S_{1}\right|>\frac{p}{2}$. Then, $S_{1}$ contains two adjacent vertices. Let $v_{i}$ and $v_{j}$ be two adjacent vertices in $S_{1}$. Clearly, $S_{1}-\left\{v_{i}\right\}$ or $S_{2}=\left\{v_{j}\right\}$ is a geodetic global dominating set of $C_{2 n}$, which is a contradiction. Therefore, $\bar{\gamma}_{g}^{+}\left(C_{2 n}\right) \leq\left\lfloor\frac{p}{2}\right\rfloor$. Hence, $\bar{\gamma}_{g}^{+}\left(C_{2 n}\right)=\left\lfloor\frac{p}{2}\right\rfloor$.

Case (ii) $p$ is odd. Let $p=2 n+1$. It is easy to verified that $S=$ $\left\{v_{1}, v_{3}, v_{5}, \ldots, v_{2 n-3}, v_{2 n-1}\right\}$ is a minimal geodetic dominating set of $C_{2 n+1}$ such that $|S|=n=\frac{p-1}{2}=\left\lfloor\frac{p}{2}\right\rfloor$. By the similar argument as in case (i), we show that $S$ is of maximal cardinality in $C_{2 n+1}$. Therefore, $\bar{\gamma}_{g}^{+}\left(C_{2 n+1}\right)=$ $\left\lfloor\frac{p}{2}\right\rfloor$. Hence, both the cases, $\bar{\gamma}_{g}^{+}\left(C_{p}\right)=\left\lfloor\frac{p}{2}\right\rfloor, p \geq 6$.

Theorem 3.20. For the wheel graph $W_{p}(p \geq 6), \bar{\gamma}_{g}^{+}\left(W_{p}\right)=\left\lceil\frac{2 p+1}{3}\right\rceil$.

Proof. Let $p \geq 6$ and $V\left(W_{p}\right)=\left\{v, v_{1}, v_{2}, . ., v_{p-1}\right\}$, where $\operatorname{deg}\left(v_{i}\right)=3$ for $1 \leq i \leq p-1$ and $\operatorname{deg}(v)=p-1$. By theorem 2.2, every geodetic global dominating set contains $\{v\}$. If $p \equiv 0(\bmod 3)$ or $p \equiv 1(\bmod$ 3) or $p \equiv 2(\bmod 3), S=\left\{v, v_{1}, v_{2}, v_{4}, v_{5}, \ldots, v_{p-5}, v_{p-4}, v_{p-2}\right\}$ or $S=$ $\left\{v, v_{1}, v_{2}, v_{4}, v_{5}, \ldots, v_{p-7}, v_{p-5}, v_{p-3}, v_{p-2}\right\}$ or

$S=\left\{v, v_{1}, v_{2}, v_{4}, v_{5}, \ldots, v_{p-7}, v_{p-6}, v_{p-4}, v_{p-2}\right\}$ is a geodetic global dominating set of $W_{p}$, respectively. And so, $|S|=\left\lceil\frac{2 p+1}{3}\right\rceil$. Let $S^{\prime}=S-\{x\}$, where $x \in S$. If $x=v$, then $S^{\prime}$ does not dominates $\overline{W_{p}}$ and so $S^{\prime}$ is not a global dominating set of $W_{p}$. If $x=v_{i}$, for $1 \leq i \leq p-1$, then $I\left[S^{\prime}\right]=V\left(W_{p}\right)$ and so $S^{\prime}$ is not a geodetic set of $W_{p}$. Therefore, $S$ is a minimal geodetic global dominating set of $W_{p}$, and so $\bar{\gamma}_{g}^{+}\left(W_{p}\right) \geq|S|$. Moreover, there does 
not exists a minimal geodetic global dominating set $S_{1}$ with $\left|S_{1}\right|>|S|$, we conclude that $\bar{\gamma}_{g}^{+}\left(W_{p}\right)=|S|=\left\lceil\frac{2 p+1}{3}\right\rceil$

Theorem 3.21. For the fan graph $F_{p}(p \geq 5), \bar{\gamma}_{g}^{+}\left(F_{p}\right)=\left\lceil\frac{2 p+1}{3}\right\rceil$.

Proof. Let $p \geq 5$ and $V\left(F_{p}\right)=\left\{v, v_{1}, v_{2}, \ldots, v_{p-1}\right\}$, where $\operatorname{deg}\left(v_{1}\right)=$ $\operatorname{deg}\left(v_{p-1}\right)=2, \operatorname{deg}(v)=p-1$ and $\operatorname{deg}\left(v_{i}\right)=3$ for $2 \leq i \leq p-2$. It is clear that $\left\{v_{1}, v_{p-1}\right\}$ be the set of extreme vertices of $F_{p}$ and $\{v\}$ is a set of full vertex in $F_{p}$. Therefore, by Theorem 2.1 and Theorem 2.2, every geodetic global dominating set contains $\left\{v_{1}, v_{p-1}, v\right\}$. If $p \equiv 0(\bmod 3)$ or $p \equiv 1(\bmod$ $3)$ or $p \equiv 2(\bmod 3)$, then $S=\left\{v, v_{1}, v_{2}, v_{4}, v_{5}, \ldots, v_{p-5}, v_{p-4}, v_{p-2}, v_{p-1}\right\}$ or $S=\left\{v, v_{1}, v_{2}, v_{4}, v_{5}, \ldots, v_{p-5}, v_{p-4}, v_{p-3}, v_{p-1}\right\}$ or $S=\left\{v, v_{1}, v_{2}, v_{4}, v_{5}, \ldots, v_{p-4}, v_{p-3}, v_{p-1}\right\}$ is a geodetic global dominating set of $F_{p}$, respectively and so $|S|=\left\lceil\frac{2 p+1}{3}\right\rceil$. As a similar discussion in Theorem 3.21 shows that $S$ is a $\bar{\gamma}_{g}^{+}$-set of $F_{p}$ and so $\bar{\gamma}_{g}^{+}\left(F_{p}\right)=\left\lceil\frac{2 p+1}{3}\right\rceil$.

Observation 3.22. Let $G=(V, E)$ be a connected graph of order $p \geq 2$ and let $S \subseteq V(G)$.

(i) If $S$ is a minimal geodetic set of $G$ which is also a global dominating set of $G$, then $S$ is a minimal geodetic global dominating set of $G$.

(ii) If $S$ is a minimal global dominating set of $G$ which is also a geodetic set of $G$, then $S$ is a minimal geodetic global dominating set of $G$.

Observation 3.23. For any connected graph $G, \gamma_{g}^{+}(G) \leq \bar{\gamma}_{g}^{+}(G)$.

Theorem 3.24. Let $S$ be a $\gamma_{g}^{+}$-set of a connected graph $G$. Then $S$ is a $\bar{\gamma}_{g}^{+}$-set of $G$ if and only if $S$ is a dominating set in $\bar{G}$.

Proof. This follows directly from the definition.

To aid in our discussion throughout this paper, we define a set $L_{S}$ as follows.

Definition 3.25. Let $G$ be a connected graph with a subset $S \subset V$ in $G$ then $L_{S}(G)$ be the set of all vertices $v$ in $V-S$ such that $v$ dominates $S$ in $G$; that is, $L_{S}(G)=\{v \in V-S \mid S \subseteq N(v)\}$. In otherwords, each vertex in $L_{S}$ is adjacent to every vertex in $S$.

Theorem 3.26. Let $G$ be a connected graph and $S$ be a $\gamma_{g}^{+}$-set of $G$. If $L_{S}=\phi$ and $S^{\prime}$ be a minimal dominating set in $\left\langle\overline{L_{S}}>\right.$, then $S \cup S^{\prime}$ is a minimal geodetic global dominating set of $G$. 
Proof. Let $L_{S}=\phi$ and let $S^{\prime}$ be a minimal dominating set in $\left\langle\overline{L_{S}}>\right.$. Since $S$ is a $\gamma_{g}^{+}$-set of $G, S \cup S^{\prime}$ dominates $\bar{G}$. It follows that $S \cup S^{\prime}$ is a geodetic global dominating set of $G$. We show that $S \cup S^{\prime}$ is a minimal geodetic global dominating set of $G$. Suppose there exists a proper subset $W$ of $S \cup S^{\prime}$ such that $W$ is a geodetic global dominating set of $G$. Then we have the following cases:

Case (i) $W \cap S=\phi$ or $W \cap S^{\prime}=\phi$. If $W \cap S=\phi$, then $W \subset S^{\prime}$. Since $W$ is a global dominating set of $G, W$ dominates $\left\langle\overline{L_{S}}>\right.$. It follows that $S^{\prime}$ is not a minimal dominating set in $\left\langle\overline{L_{S}}>\right.$. Suppose $W \cap S^{\prime}=\phi$ then $W \subset S$. Since $W$ is both geodetic and global dominating set of $G, W$ is a geodetic global dominating set of $G$. Thus $S$ is not a minimal geodetic global dominating set of $G$.

Case (ii) $S \cap W=\phi$ and $S^{\prime} \cap W=\phi$. Since $S \cap S^{\prime}=\phi, W=S$ and $W=S^{\prime}$. If $W=S$, choose a vertex $v_{i} \in S$ such that $v_{i} \notin W$. Then the vertex $v_{i}$ does not lies on any geodesic joining pair of vertices of $W$, implies that $W$ is not a geodetic global dominating set of $G$. If $W=S^{\prime}$, there exits a vertex $y_{i} \in S^{\prime}$ such that $y_{i} \notin W$. Then $y_{i} \notin N[W]$ in $\bar{G}$ implies that $W$ is not a geodetic global dominating set of $G$. Thus, both the ways we obtained a contradiction. Hence, $S \cup S^{\prime}$ is a minimal geodetic global dominating set of $G$.

Theorem 3.27. Let $G$ be a connected graph and $S$ be a $\gamma_{g}^{+}$-set of $G$. Then, $\bar{\gamma}_{g}^{+}(G) \leq \gamma_{g}^{+}(G)+\left|L_{S}\right|$.

Proof. Let $S$ be a minimal geodetic dominating set of $G$ such that $\gamma_{g}^{+}(G)=|S|$. If $S$ dominates $\bar{G}$, then by Theorem $3.25, S$ is a $\bar{\gamma}_{g}^{+}$-set of $G$ and so $\bar{\gamma}_{g}^{+}(G)=|S|=\gamma_{g}^{+}(G)$. Now, assume that $S$ does not dominates $\bar{G}$. Then, there exist vertices in $V-S$, which is adjacent to every vertices in $S$. This shows that $L_{S}=\phi$. Let $S^{\prime}$ be a minimal dominating set in $\left\langle\overline{L_{S}}>\right.$ with maximum cardinality. Then by Theorem 3.27, $S \cup S^{\prime}$ is a minimal geodetic global dominating set in $G$. We note that $S$ and $S^{\prime}$ is of maximum cardinality. Since $S \cap S^{\prime}=\phi, S \cup S^{\prime}$ is a $\bar{\gamma}_{g}^{+}$-set of $G$ and so $\bar{\gamma}_{g}^{+}(G)=\left|S \cup S^{\prime}\right|$. Since $\left|S^{\prime}\right| \leq\left|L_{S}\right|$, we conclude that $\bar{\gamma}_{g}^{+}(G) \leq \gamma_{g}^{+}(G)+\left|L_{S}\right|$.

Theorem 3.28. Let $G$ be a connected graph. If $S$ is a $\gamma_{g}^{+}$-set of $G$, then (i) $\bar{\gamma}_{g}^{+}(G)=\gamma_{g}^{+}(G)$ if and only if $L_{S}=\phi$.

(ii) $\bar{\gamma}_{g}^{+}(G)=\gamma_{g}^{+}(G)+1$ if and only if $\left\langle L_{S}>\right.$ is totally disconnected.

(iii) $\bar{\gamma}_{g}^{+}(G)=\gamma_{g}^{+}(G)+\left|L_{S}\right|$ if and only if $\left\langle L_{S}>\right.$ is complete. 
Proof. Let $G$ be a connected graph and $S$ be a $\gamma_{g}^{+}$-set of G.

(i) First assume $L_{S}=\phi$. Then by Theorem 3.28, $\bar{\gamma}_{g}^{+}(G) \leq \gamma_{g}^{+}(G)$. By Observation 3.24, we conclude that $\bar{\gamma}_{g}^{+}(G)=\gamma_{g}^{+}(G)$. Now assume $\bar{\gamma}_{g}^{+}(G)=\gamma_{g}^{+}(G)$. Let $S$ be a $\gamma_{g}^{+}$-set of $G$ which is also a $\bar{\gamma}_{g}^{+}$-set of $G$. Therefore by Theorem $3.25, S$ is a dominating set of $\bar{G}$, implies no vertex in $V-S$ is adjacent to every vertex in $S$. That is, $S N(v)$ for every $v$ in $V-S$. Hence, $L_{S}=\phi$.

(ii) Assume $\left\langle L_{S}>\right.$ is totally disconnected. Let $x \in V\left(<L_{S}>\right)$.Then, $S \cup\{x\}$ is a $\bar{\gamma}_{g}^{+}$-set of $G$ and so $\bar{\gamma}_{g}^{+}(G)=|S \cup\{x\}|=\gamma_{g}^{+}(G)+1$. Conversely, assume $\bar{\gamma}_{g}^{+}(G)=\gamma_{g}^{+}(G)+1$. By (i) $L_{S}=\phi$. To prove $\left\langle L_{S}\right\rangle$ is totally disconnected. Suppose $\left\langle L_{S}\right\rangle$ contains a vertex $x$ such that $x$ is adjacent to at least one vertex $y$ in $V\left(<L_{S}>\right)$. This shows every minimal geodetic global dominating set contains $S \cup\{x, y\}$, implies $\bar{\gamma}_{g}^{+}(G) \geq \gamma_{g}^{+}(G)+2$. which is a contradiction. Hence $\left\langle L_{S}\right\rangle$ is totally disconnected.

(iii) Assume $\left\langle L_{S}>\right.$ is complete. Then $V\left(<L_{S}>\right)$ is a minimum dominating set in $\left\langle\overline{L_{S}}>\right.$. Since $S \cap L_{S}=\phi, S \cup L_{S}$ is a minimal geodetic global dominating set of $G$ and so $\bar{\gamma}_{g}^{+}(G) \geq\left|S \cup L_{S}\right|=\gamma_{g}^{+}(G)+\left|L_{S}\right|$. By Theorem 3.28, we conclude $\bar{\gamma}_{g}^{+}(G)=\gamma_{g}^{+}(G)+\left|L_{S}\right|$. Conversely, assume $\bar{\gamma}_{g}^{+}(G)=\gamma_{g}^{+}(G)+\left|L_{S}\right|$. To prove $\left\langle L_{S}>\right.$ is complete. Suppose $\left\langle L_{S}>\right.$ is non-complete. By (i) $L_{S}=\phi$. There exists a vertex $x$ in $L_{S}$, which is non-adjacent to a vertex $y$ in $L_{S}$. It follows that $S \cup\left(L_{S}-\{x\}\right)$ is a $\bar{\gamma}_{g}^{+}$-set of $G$ and so $\bar{\gamma}_{g}^{+}(G)=\left|S \cup\left(L_{S}-\{x\}\right)\right|<\bar{\gamma}_{g}^{+}(G)+\left|L_{S}\right|$, a contradiction to our hypothesis. Hence $<L_{S}>$ is complete.

Theorem 3.29. For every pair of integers $a$ and $b$ with $2 \leq a \leq b$, there exists a connected graph $G$ such that $\gamma_{g}^{+}(G)=a$ and $\bar{\gamma}_{g}^{+}(G)=b$.

Proof. If $a=b$, let $G$ be a complete graph of order $a$. Then by Corollary $3.12, \gamma_{g}^{+}(G)=\bar{\gamma}_{g}^{+}(G)=a$. Now, let $a<b$. We consider two cases:

Case (i) Let $b=a+1$. Consider the graph $G$ obtained from the path graph on three vertices $P_{3}: x, y, z$ by adding new vertices $v_{1}, v_{2}, \ldots, v_{a-1}$ and join $v_{1}, v_{2}, \ldots, v_{a-1}$ to both $x$ and $z$. This is shown in Figure 3.5. Let $S=\left\{y, v_{1}, v_{2}, \ldots, v_{a-1}\right\}$. It is easy to observe that $S$ or $\{x, z\}$ are the only minimal geodetic dominating sets of $G$ and so $\gamma_{g}^{+}(G)=|S|=a$. Now, let $S_{1}=S \cup\{x\}$ or $S_{1}=S \cup\{z\}$. It is clear that $S_{1}$ is a geodetic global 
dominating set of $G$. Now, we show that $S_{1}$ is a minimal geodetic global dominating set of $G$. Suppose on the contrary, that $S_{1}$ is not a minimal geodetic global dominating set of $G$. Then, there is a proper subset $S^{\prime}$ of $S_{1}$ such that $S^{\prime}$ is a geodetic global dominating set of $G$.

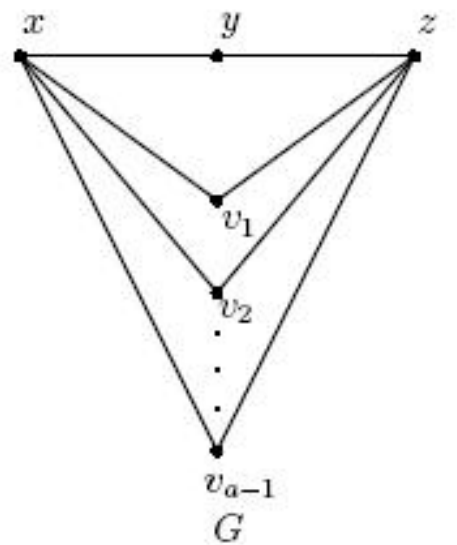

Figure 3.5

Let $v \in S_{1}$ and $v \notin S^{\prime}$. If $v=x$ or $v=z$, then $S^{\prime}$ does not dominates $\bar{G}$. If $v=v_{i}$ for some $i=1,2, \ldots, a-1$, then $v_{i}$ does not lie on a geodesic $P$ joining any pair of vertices in $S^{\prime}$ and so that $S^{\prime}$ is not a geodetic global dominating set of $G$, which is a contradiction. Thus, $S_{1}$ is a minimal geodetic global dominating set of $G$ and so $\bar{\gamma}_{g}^{+}(G) \geq\left|S_{1}\right|=b$. Since the order of $G$ is $b+1$ and either $x \notin S_{1}$ or $z \notin S_{1}$, we conclude that $\bar{\gamma}_{g}^{+}(G)=b$. 


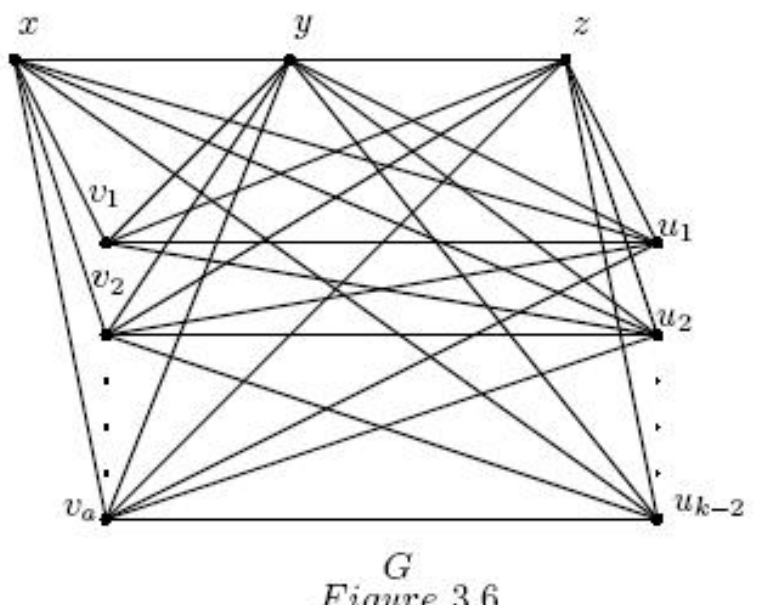

Figure 3.6

Case (ii) Let $b=a+k$, where $k \geq 2$. Consider the graph $H$ obtained from the path $P_{3}: x, y, z$ by adding new vertices $v_{1}, v_{2}, \ldots, v_{a}$ and join each $v_{i}(1 \leq i \leq a)$ with $x, y, z$. Now, we add $k-2$ new vertices $u_{1}, u_{2}, \ldots, u_{k-2}$ to the graph $H$ and join each $u_{i}(1 \leq i \leq k-2)$ to every vertices in $H$, thereby producing the graph $G$ in Figure 3.6. Then, as in case (i), $S=\left\{v_{1}, v_{2}, \ldots, v_{a}\right\}$ is a minimal geodetic dominating set with maximum cardinality and $V(G)-\{x\}$ is a minimal geodetic global dominating set with maximum cardinality. Thus, $\gamma_{g}^{+}(G)=a$ and $\bar{\gamma}_{g}^{+}(G)=b$.

Corollary 3.30. For every pair of integers $a$ and $b$ with $2 \leq a<b$, the smallest possible order of a connected graph $G$ for which $\gamma_{g}^{+}(G)=a$ and $\bar{\gamma}_{g}^{+}(G)=b$ is $b+1$.

Theorem 3.31. For any three integers $a, b, p$ with $3 \leq a \leq b<p$, there exists a connected graph $G$ of order $p$, such that $\bar{\gamma}_{g}(G)=a$ and $\bar{\gamma}_{g}^{+}(G)=b$.

Proof. If $p=b+1$, then let $a=3+k$ and $b=r+k$, where $k \geq 0$ and $r \geq 3$. Assume $k=0$. Consider the graph $G=K_{2, r-1}$ with partition $X=\left\{x_{1}, x_{2}\right\}$ and $Y=\left\{y_{1}, y_{2}, \ldots, y_{r-1}\right\}$ as shown in Figure 3.7. By Theorem 2.5, $\overline{\gamma_{g}}(G)=\min \{X, Y\}+1=3$. Also by Theorem 3.18, $\bar{\gamma}_{g}^{+}(G)=\max \{X, Y\}+1=r$. Therefore, in this case $\overline{\gamma_{g}}(G)=3$ and $\bar{\gamma}_{g}^{+}(G)=r$. 


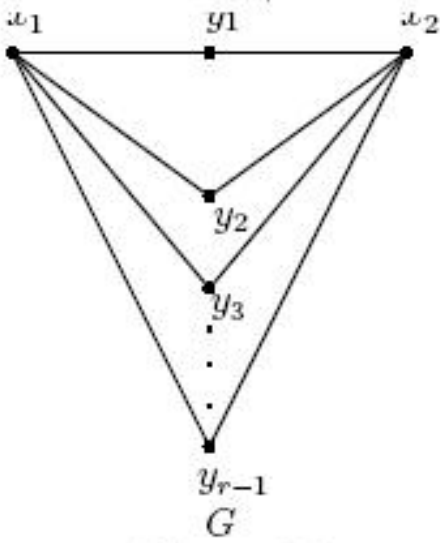

Figure 3.7

Now, suppose that $k \geq 1$. Let $H=K_{2}+\overline{K_{k}}$ with $V\left(K_{2}\right)=\{y, z\}$ and $V\left(\overline{K_{k}}\right)=\left\{z_{1}, z_{2}, \ldots, z_{k}\right\}$. Let $G$ be the connected graph obtained by taking join of $K_{1, r-2}$ (with partite sets $\{x\}$ and $\left.\left\{y_{1}, y_{2}, \ldots, y_{r-2}\right\}\right)$ and $H$ and joining each $y_{i}(1 \leq i \leq r-2)$ with $y$ and $z$. Also add a new edge $x z$ in $G$ and is shown in Figure 3.8 .

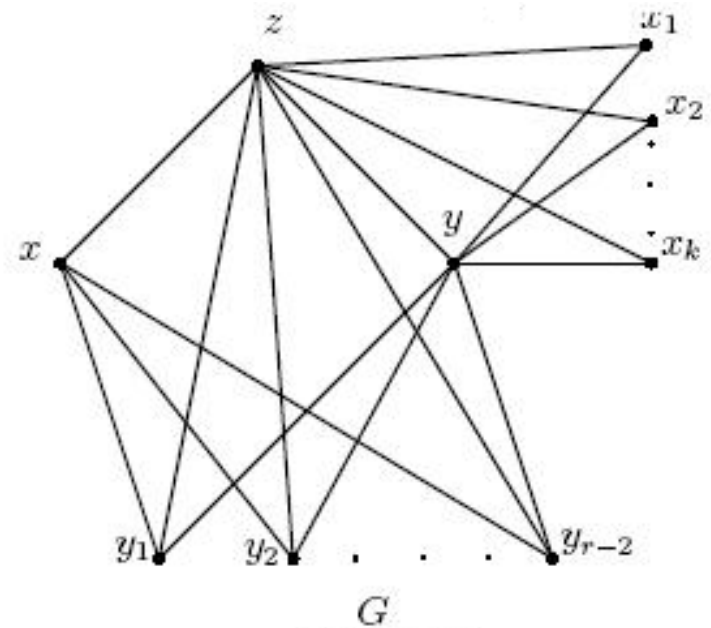

Figure 3.8

It is clear that $\operatorname{diam}(G)=2$ and $G$ is a connected graph of order $b+1$. Also, $\operatorname{Ext}(G)=\left\{x_{1}, x_{2}, \ldots, x_{k}\right\}$ and $F_{x}(G)=\{z\}$. The set $S=$ $\operatorname{Ext}(G) \cup F_{x}(G)$ is not a geodetic global dominating set in $G$. We note that $S \cup\{x, y\}$ is a minimum geodetic global dominating set in $G$ and so $\bar{\gamma}_{g}(G)=|S \cup\{x, y\}|=3+k=a$. Since $S$ is always contained in a geodetic 
global dominating set in $G$, that $S \cup\left\{y_{1}, y_{2}, \ldots, y_{r-2}\right\}$ is a minimal geodetic global dominating set of $G$ and so $\bar{\gamma}_{g}^{+}(G) \geq b$. Since the order of $G$ is $b+1$,by Theorem 3.11, it follows that $\bar{\gamma}_{g}^{+}(G)=b$. Suppose that $p=b+2$. Let $a=3+k$ and $b=r+k$, where $r \geq 3$ and $k \geq 0$. Let $G$ be the graph obtained from $K_{3, r-2}$ (with particle sets $\{x, y, z\}$ and $\left\{y_{1}, y_{2}, \ldots, y_{r-2}\right\}$ ) and $K_{1, k+1}$ (with partite sets $\{z\}$ and $\left\{x_{1}, x_{2}, \ldots, x_{k+1}\right\}$ ) using $z$ as a common vertex. This is the graph $G$ as shown in Figure 3.9

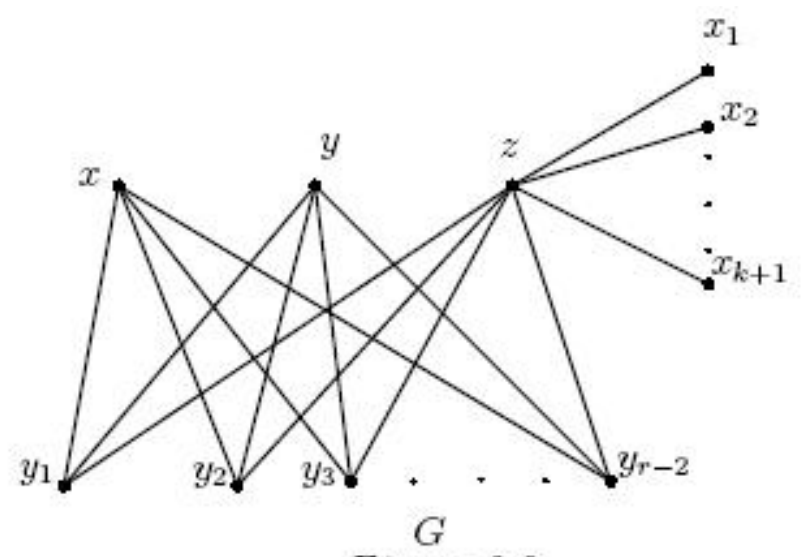

Figure 3.9

Let $S=\left\{x_{1}, x_{2}, \ldots, x_{k+1}\right\}$ be the set of all extreme vertices of $G$. By Theorem 2.1, every geodetic global dominating set contains $S$. It is clear that $S$ is not geodetic global dominating set in $G$. But $S^{\prime}=S \cup\{x, y\}$ is a minimum geodetic global dominating set of $G$ and so $\overline{\gamma_{g}}(G)=|S \cup\{x, y\}|=$ $a$. Let $S_{1}=S \cup\left\{y_{1}, y_{2}, \ldots, y_{r-2}\right\}$. It is clear that $S_{1}$ is not an geodetic global dominating set in $G$. Clearly, $S_{2}=S_{1} \cup\{z\}$ is a geodetic global dominating set of $G$. Now, we show that $S_{2}$ is a minimal geodetic global dominating set in $G$. Suppose on the contrary, there exists a proper subset $W$ of $S_{2}$ such that $W$ is a geodetic global dominating set in $G$. Chose a vertex $v \in S_{2}$ such that $v \notin W$. Clearly $v=x_{i}$ for $1 \leq i \leq k+1$. Suppose $v=y_{i}$ for 
some $i \in\{1,2, \ldots, r-2\}$. Then the vertex $v$ dose not lies on any geodesic pair of vertices of $W$, which is a contradiction. If $v=z$, then $W$ is not a global dominating set of $G$, a contradiction. Thus, $S_{2}$ is a minimal geodetic global dominating set of $G$ and so $\bar{\gamma}_{g}^{+}(G) \geq\left|S_{2}\right|=b$. Moreover, any minimal geodetic global dominating set contains the set $\{x, y\}$ coincides $S^{\prime}$. Therefore every minimal geodetic global dominating set other than $S^{\prime}$ contains either $x$ or $y$ or $z$. Hence, $\bar{\gamma}_{g}^{+}(G)=b$. Suppose that $p=b+l$, where $l \geq 3$. Let $a=3+k$ and $b=r+k, r \geq 3$ and $k \geq 0$. Consider the graph obtained as follows:

(i) Take a copy of $K_{1, k+1}$ with $(k+1)$-pendent vertices $w, z_{1}, z_{2}, \ldots, z_{k}$ and the support vertex $z$.

(ii) Take a copy of $K_{2, r-1}$ with partite sets $\{x, y\}$ and $\left\{y_{1}, y_{2}, . ., y_{r-1}\right\}$.

(iii) Add a new edge $y w$.

Let $G$ be the graph in Figure 3.10 is obtained from $H$ and a copy of complete graph $K_{p-b-2}$ with $(p-b-2)$ vertices $x_{1}, x_{2}, \ldots, x_{p-b-2}$ and joining each $x_{i}(1 \leq i \leq p-b-2)$ with $x, y$ and $z$.

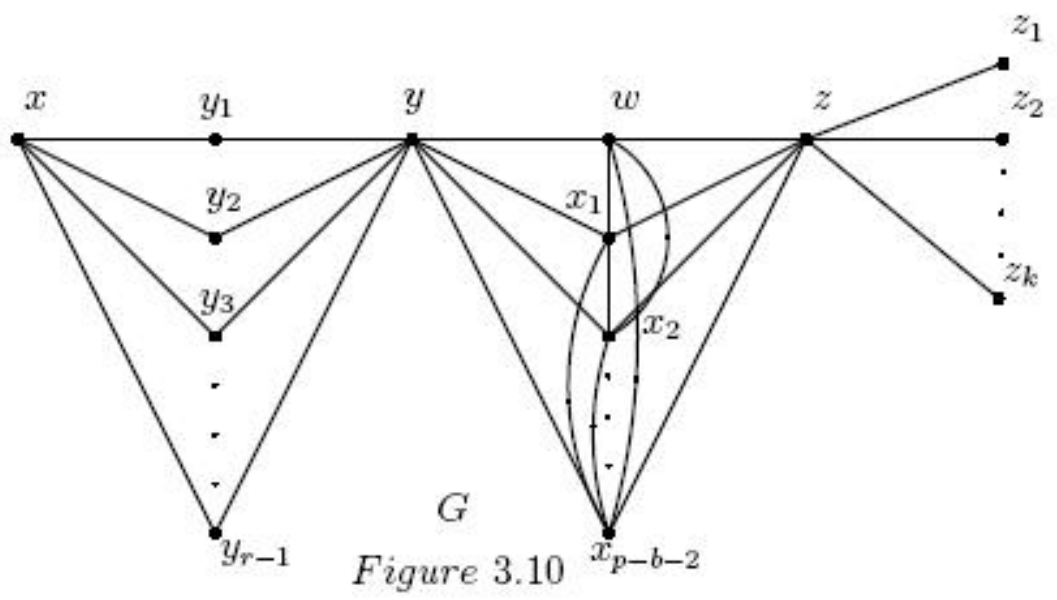


Let $S=\left\{z_{1}, z_{2}, \ldots, z_{k}\right\}$ be the set all extreme vertices of $G$. It is easily to verified that $S$ is not a geodetic global dominating set in $G$. Clearly that $S \cup\{x, y\}$ is a $\bar{\gamma}_{g}$-set of $G$ and so $\bar{\gamma}_{g}(G)=|S|+2=k+2=a$. Also, it is easily seen that $S \cup\left\{y_{1}, y_{2}, \ldots, y_{r-1}, z\right\}$ is a $\bar{\gamma}_{g}^{+}$-set of $G$. In this case, $\bar{\gamma}_{g}(G)=a$ and $\bar{\gamma}_{g}^{+}(G)=r+k=b$.

\section{Acknowledgment}

The authors are thankful to the referee whose valuable suggestions for the revised verson of this paper.

\section{References}

[1] F. Buckley and F. Harary, Distance in graphs. Redwood City, CA: Addison-Wesley, 1990.

[2] G. Chartrand, F. Harary, and P.Chang, "On the geodetic number of a graph", Networks, vol. 39, no.1, pp. 1-6, Jan. 2002, doi: 10.1002/ net.10007

[3] W. J. Desormeaux, P. E. Gibson, and T. W. Haynes, "Bounds on the global domination number", Quaestiones mathematicae, vol. 38, no. 4, pp. 563-572, Jun. 2015, doi: 10.2989/ 16073606.2014.981728

[4] H. Escuadro, R. Gera, A. Hansberg, N. Jafari Rad, and L. Volkmann, "Geodetic domination in graphs", Journal of combinatorial mathematics and combinatorial computing, vol. 77, pp. 88-101, May 2011. [On line]. Available: https:/ / bit.ly/ 3p6DuWZ

[5] T. W. Haynes, P. J. Slater, and S. T. Hedetniemi, Fundamentals of domination in graphs. Boca Raton, CA: CRC Press, 1998, doi: $10.1201 / 9781482246582$

[6] H. M. Nuenay and F. P. Jamil, "On minimal geodetic domination in graphs", Discussiones mathematicae graph theory, vol. 35, no. 3, pp. 403-418, Jul. 2015, doi: 10.7151/ dmgt.1803

[7] S. R. Chellathurai and L. Xaviour, "Geodetic global domination in graphs", International journal of mathematical archive, vol. 9, no. 4, pp. 29-36, 2018. [On line]. Available: https:/ / bit.ly/38mtIKv

[8] E. Sampath Kumar, "The global domination number of a graph", Journal of mathematical and physical sciences, vol. 23, no. 5, pp. 377-385, 1989. [On line]. Available: https:/ / bit.ly/ 3kbwpR6

[9] D. B. West, Introduction to graph theory, 2nd ed. Upper Saddle River, NJ: Prentice-Hall, 2001. 